\title{
Aerodynamics of an isolated ski jumping ski
}

\author{
Mikko Virmavirta ${ }^{1}\left[\right.$ ] Juha Kivekäs ${ }^{2}$
}

Published online: 8 February 2019

(c) The Author(s) 2019

\begin{abstract}
A single isolated ski was suspended from a six-component wind tunnel balance and three angles, the angle of attack, the yaw angle and the edge angle were adjustable during the test. Increasing yaw angle from 0 to $15^{\circ}$ increased the lift coefficient $C_{L}$ from 0.42 to 0.90 at edge angle $0^{\circ}$ and from 0.70 to 0.87 at edge angle $10^{\circ}$, respectively. Increasing yaw angle also increased the sensitivity of the ski to changes in edge angle, i.e., increasing the edge angle $\left(20^{\circ}-45^{\circ}\right)$ decreased the $C_{L}$ and the ratio $C_{L}^{2} / C_{D}$ with large yaw angles. However, to maximize the lift-to-drag ratio with a typical angle of attack of $30^{\circ}$ in ski jumping, it may be reasonable to have an edge angle of $5^{\circ}-10^{\circ}$ on skis as the ratio $C_{L}^{2} / C_{D}$ increased from 1.24 to 1.35 when edge angle increased from $0^{\circ}$ to $10^{\circ}$.
\end{abstract}

\section{Introduction}

In ski jumping the flight style changed in the early 1990s, when $\mathrm{V}$-style replaced the traditional style in which skis were held parallel and close to each other. Swedish ski jumper Jan Boklöv was one of the first jumpers to use V-style, and his success (overall World Cup winner 1988-1989) attracted scientific attention to this new flight style. As there was an urgent need among coaches and others in ski jumping to enhance their understanding of the merits of this new flight style, several studies were conducted during those early years of V-style jumping (for review see [1]). The first and probably the most important study of V-style was published by Mahnke and Hochmuth [2]. They performed series of wind-tunnel experiments while investigating the benefits of the V-style. The results showed that all V-style ski positions were better than the traditional ones and improved the aerodynamic quality of the jumper/skis system. Almost all studies of V-style jumping have dealt with the $V$ angle of skis (2 times the yaw angle used in the present study) at

Electronic supplementary material The online version of this article (https://doi.org/10.1007/s12283-019-0298-1) contains supplementary material, which is available to authorized users.

Mikko Virmavirta

mikko.virmavirta@jyu.fi

1 Faculty of Sport and Health Sciences, Biology of Physical Activity, University of Jyväskylä, P.O. Box 35, 40014 Jyväskylä, Finland

2 Arteform Oy, Espoo, Finland different ski angles of attack (AoA). The optimum $V$ angle has typically been found to be a constant value of around $25^{\circ}-30^{\circ}$. Seo et al. [3] suggested that to reduce drag in the early flight phase, the optimum $V$ angle should be around $26^{\circ}$ to maximize lift during the rest of a jump. However, it may be practically impossible for a jumper to precisely control the $V$ angle in the early phases of a jump. In the literature there is information on yaw $\left(\sim 15^{\circ}-20^{\circ}\right)$ and AoA angles $\left(\sim 25^{\circ}-40^{\circ}\right)$ used by ski jumpers during most of the flight phase (e.g., [4]), but less about roll of the ski around its longitudinal axis which corresponds to the edge angle described in the present study. Bessone et al. [5] used inertial sensors to measure the edge angle of both skis during the flight phase of two jumpers. The angle ranged from $0^{\circ}$ (flat ski) at the release instant from the take-off table to $30^{\circ}-40^{\circ}$ at mid-flight. Some jumpers may have an unbalanced ski position, with different edge angles between the two skis, as was probably the case for one of the jumpers in the study of Bessone et al., who showed a $10^{\circ}-20^{\circ}$ difference between the edge angles of the left and right skis.

One study where aerodynamics of a ski jumper and skis were analysed separately was performed by Nørstrud and Øye [6] utilizing Computational Fluid Dynamics (CFD, non-viscous Euler computations). They found that the maximum simulated lift on skis and jumper without skis showed similar trends, whereby the optimum $V$ angle was around $30^{\circ}$. The highest aerodynamic lift coefficient $\left(C_{L}\right)$ of 0.440 was found at $25^{\circ}$ when the distribution of the aerodynamic characteristics was studied along a single ski axis. The flow visualization showed an important detail of the ski jumping 
ski, as the drag producing vortex leaving from the outer side of the ski tip was clearly seen. This observation led to a proposal for a new ski design. Air flow interaction between skis and the jumper is not fully understood. In the article of Nørstrud and Øye [6], the simplified geometric ski jumper with flow visualization (CFD) showed how the streamlines behave around a jumper and skis. From this the interaction can be visually estimated, but true flow interaction was not studied.

In V-style jumping, where the skis and the jumper's legs are spread out (Fig. 1a), the skis usually roll around their longitudinal axis as described above. Cutter [7] conducted comprehensive tests with a scaled ski jumper model (1:5.5) in the subsonic wind tunnel at the United States Air Force
Academy Aeronautics. Toe out angle (corresponding to the yaw angle of the present study) and ankle angle (bottom of feet inward, see ankle joint inversion in Fig. 5) were considered to be key in forming the $\mathrm{V}$ configuration. In this study, a $V$ angle of $22.5^{\circ}$ with an ankle angle of $20^{\circ}$ (which was considered the maximum feasible for a ski jumper to keep the skis more flat against the direction of motion) provided the best lift-to-drag $(L / D)$ ratio of 1.55 . A significant improvement in $L / D$ was found when ankle angle changed from $0^{\circ}$ to $20^{\circ}$, especially in the lower angle of attack region (AoA). As the skis became more effective, the trim AoA (balanced pitching moment) reduced as well. This compensatory action at the ankle joint has not subsequently been studied in detail. Around 2010, ski jumpers started to use curved sticks at the
Fig. 1 a Typical V-style jumping showing how skis turn on their inner edge when the skis and the jumper's legs are spread out (photo: Patrick Forsblom). b Examples of curved sticks used at the back part of ski bindings. From the figures on the right it is evident that this mechanism requires the shoe sole to twist when the heel part of the jumping shoe is raised from the heel block and the fixation point (white clip on the left) follows the curved stick
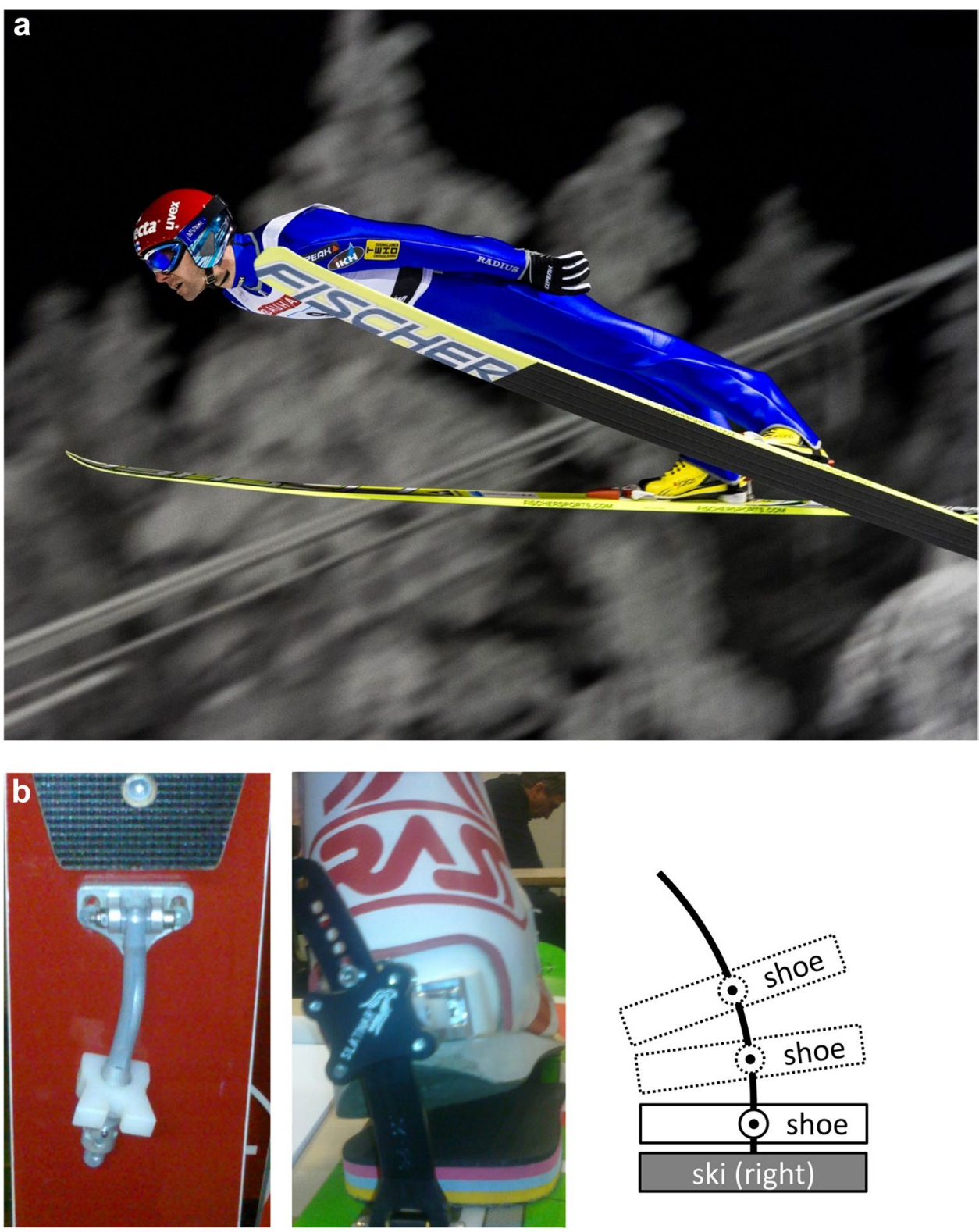
back part of their ski bindings (Fig. 1b) to keep the skis more flat and improve aerodynamics of the jumper/ski system. The functionality of this curved stick is questionable since the front part of the jumping shoe must stay fixed in the front part of the binding and horizontal to the top surface of the ski [8]. Thus, for the rear part of the jumping shoe to follow the curved stick (see Fig. 1b) the shoe sole must be twisted somehow. The true effect of edge angle of skis has not been studied in detail, and therefore, the present study examined the combined effect of $\mathrm{V}$ and edge angle with different AoAs on isolated ski aerodynamics in a wind tunnel.

\section{Methods}

The tests were carried out in a Göttingen-type closed-circuit low-speed wind tunnel [9] with a cross section of $3.68 \mathrm{~m}^{2}$ and a maximum speed of $70 \mathrm{~m} \mathrm{~s}^{-1}$. A large settling chamber with a contraction ratio of 13 and a flow velocity variation in the tunnel test section (i.e., the non-uniformity of flow) of $0.12 \%$ provided a low turbulence intensity $(<0.2 \%)$ in the empty test section. A Pitot tube connected to a Rosemount pressure meter was used to derive the wind velocity from the kinetic pressure. The true flow velocity was calculated according to

$v=\sqrt{2 q \rho^{-1}}$,

where $q$ is the kinetic pressure (Pa) and $\rho$ is the air density $\left(\mathrm{kg} \mathrm{m}^{-3}\right)$. The air density, $\rho$, was calculated according to

$\rho=p / R T$,

where $p$ is the air pressure (Pa), $T$ is the air temperature $(\mathrm{K})$ and $R$ is the gas constant $\left(287.1 \mathrm{~J} \mathrm{~K}^{-1} \mathrm{~kg}^{-1}\right)$. Tests were done with a real $100 \%$ size ski jumping ski $(242 \mathrm{~cm}$, reference area $0.2662 \mathrm{~m}^{2}$ ) with a realistic speed of $28-30 \mathrm{~m} \mathrm{~s}^{-1}$. A single isolated ski was suspended from the 6-component wind tunnel balance (Fig. 2a). The angle of attack (AoA), yaw angle and edge angle (Fig. $2 \mathrm{~b}$ ) were adjustable during the test. Every test run consisted of an AoA between $0^{\circ}$ and $40^{\circ}$ (in $2^{\circ}$ steps) covering all the angles during the actual ski jump. Yaw angles of $0^{\circ}, 2^{\circ}, 5^{\circ}, 10^{\circ}, 15^{\circ}$ and $20^{\circ}$ were used, corresponding to $V$ angles between $0^{\circ}$ and $40^{\circ}$. Before each run, the edge angle was manually adjusted to $0^{\circ}, 5^{\circ}, 10^{\circ}$, $20^{\circ}, 30^{\circ}$ and $45^{\circ}$ using an inclinometer (Fig. 2b). The total number of measurements with all of the above-mentioned set-up variations was 756 .

The balance recorded all six reaction components (3D forces and moments) and the average value of ten samples during a 5-s recording period was used for the analysis. All reaction results were converted to non-dimensional lift and drag coefficients $\left(C_{L}, C_{D}\right)$ according to standard practice in aerodynamics, utilizing equations for aerodynamic lift and drag forces, $F_{L, D}=1 / 2 \rho v^{2} C_{L, D} A$, where the quantity one-half

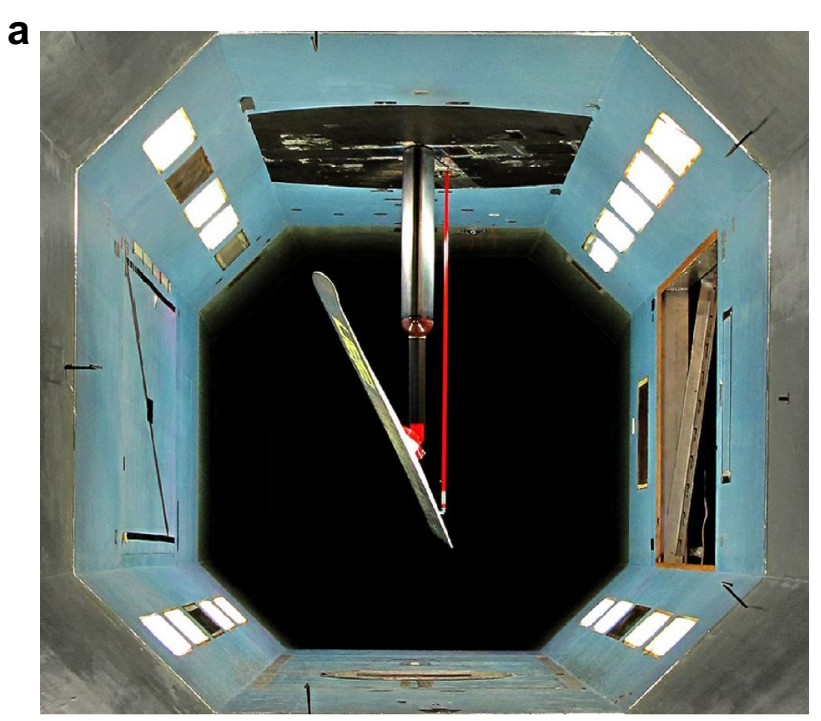

b
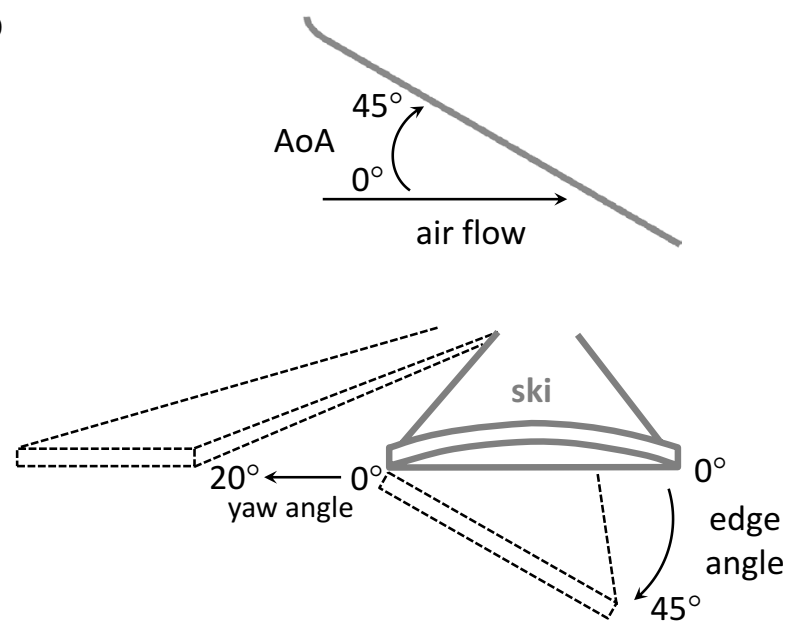

Fig. 2 a Front view of a single isolated right ski suspended from the 6-component wind tunnel balance. b Ski angles used: AoA (above), yaw and edge angles (below)

the density times the velocity squared is the kinetic pressure and $A$ is the reference area.

\section{Results}

Figure 3 a shows the effect of yaw angle on the aerodynamic lift $\left(C_{L}\right)$ with an edge angle of $0^{\circ}$ (flat ski) and increasing AoA $\left(0^{\circ}-40^{\circ}\right)$. The effect of edge angle on $C_{L}$ with a yaw angle of $15^{\circ}$ can be seen in Fig. 3b, and the relationship between $C_{L}$ and $C_{D}$ (aerodynamic polar, AoA $0^{\circ}-40^{\circ}$ ) with a yaw angle of $15^{\circ}$ (typical $30^{\circ} \mathrm{V}$ angle during most of the flight) is presented in Fig. 3c. The combined effect of yaw and edge angles with a $30^{\circ}$ AoA (typical AoA during most of the flight) on $C_{L}$ is presented in 


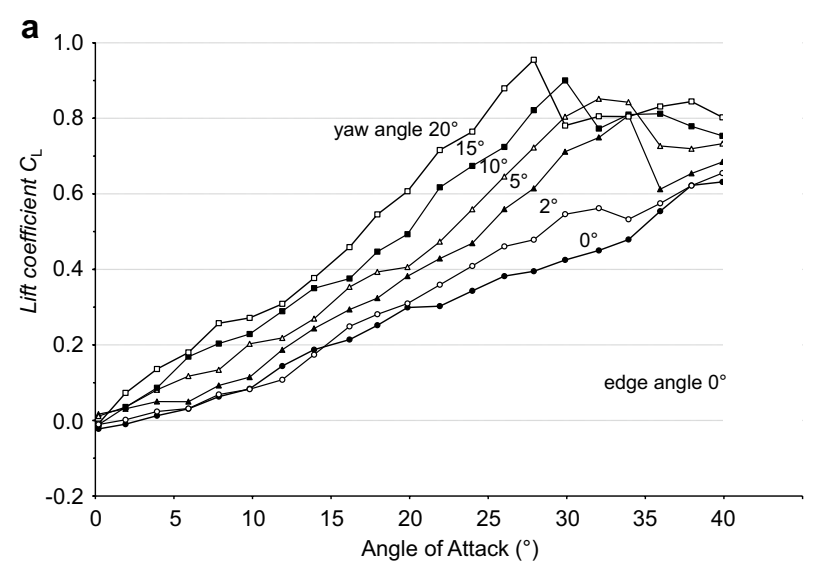

b

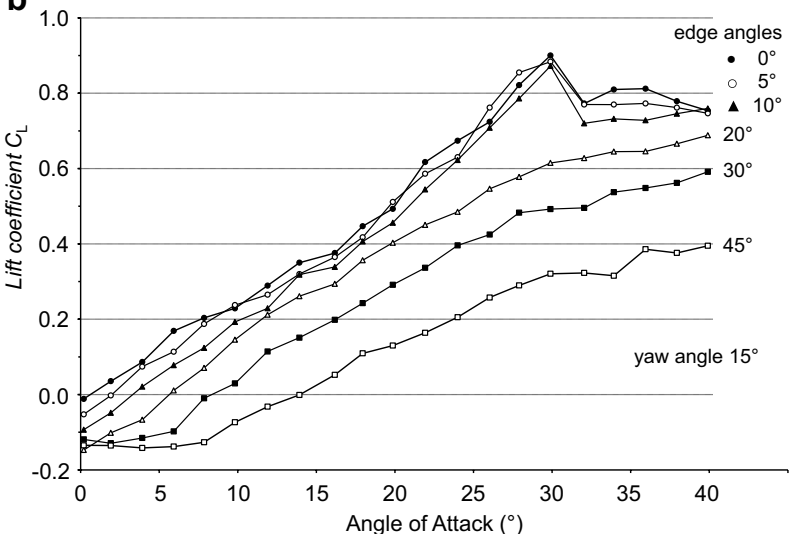

C

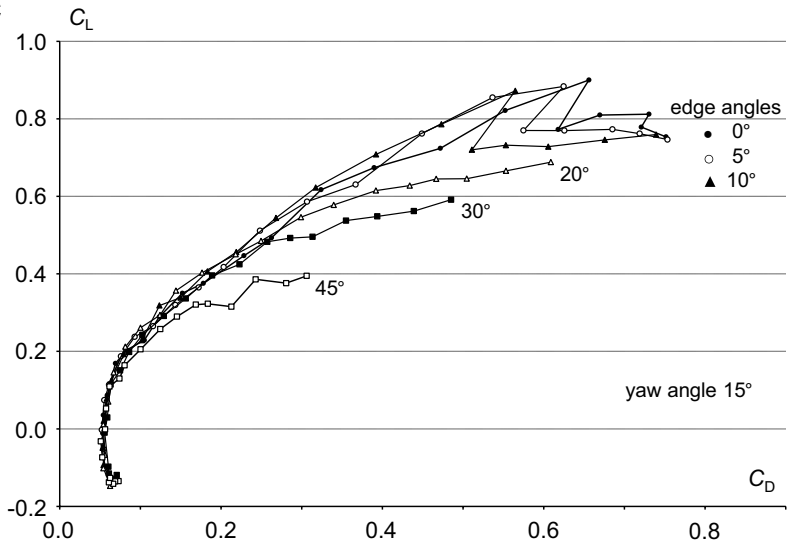

Fig. 3 a The effect of yaw angle on aerodynamic lift $\left(C_{L}\right)$ with an edge angle of $0^{\circ}$ (flat ski) and increasing AoA $\left(0^{\circ}-40^{\circ}\right)$. b The effect of edge angle on aerodynamic lift $\left(C_{L}\right)$ with a yaw angle of $15^{\circ}$ at different AoA $\left(0^{\circ}-40^{\circ}\right)$. c The effect of edge angle on aerodynamic polar (lift-to-drag ratio, $C_{L} / C_{D}$, ) with a typical yaw angle of $15^{\circ}$ (AoA between $0^{\circ}$ and $40^{\circ}$ in $2^{\circ}$ steps)

Fig. 4a, and the effect on the emphasized lift-to-drag ratio $\left(C_{L}^{2} / C_{D}\right)$ is shown in Fig. $4 \mathrm{~b}$. The supplementary video recording shows the unsteady vibration of the ski at the highest angle of attack $\left(>40^{\circ}\right)$ without any additional increase in AoA.

\section{Discussion}

The purpose of the present study was to examine the combined effect of AoA, yaw $(V)$ and edge angles of a ski jumping ski on ski aerodynamics. With all AoAs $\left(0^{\circ}-40^{\circ}\right.$, except $30^{\circ}-34^{\circ}$ ) increasing the yaw angle of an isolated ski $\left(0^{\circ}>20^{\circ}\right.$, corresponding to a $V$ angle $\left.0^{\circ}>40^{\circ}\right)$ increased the aerodynamic lift $\left(C_{L}\right)$, especially with a $0^{\circ}$ edge angle (Fig. 3a). Paradoxically, not feasible for a jumper, $C_{L}$ would probably continue to increase until the $90^{\circ}$ yaw angle, in which case the isolated ski would resemble a long, narrow wing. A long, narrow wing has a high aspect ratio (the wing's span relative to its mean chord) and therefore offers aerodynamic advantages like a better lift-todrag ratio. As seen in Fig. $3 \mathrm{a}-\mathrm{c}$, there is a $C_{L}$ ear discontinuity on the $C_{L}$ curve depending on the combined effect of yaw $\left(5^{\circ}-20^{\circ}\right)$ and edge angles $\left(0^{\circ}-10^{\circ}\right)$. This happens at higher angles of attack $\left(\sim 25^{\circ}-30^{\circ}\right)$ when maximum $C_{L}$ is reached. Ski position in the direction of air flow is a combination of the yaw and edge angles and not necessarily the AoA as it is usually described in the V-style ski plane and therefore, the cross-sectional profile of one ski may even momentarily and accidentally be at $0^{\circ}$ angle in the direction of air flow during the early flight phase when the final yaw and edge angles are being formed. These situations have been seen sometimes in ski jumping.

In the supplementary video file the ski is most likely stalling or unlikely experiencing vortex shedding at high AoA, which is, however, beyond the AoAs $\left(>40^{\circ}\right)$ used in the present study (not the discontinuity point in the curves $3 \mathrm{a}-\mathrm{c}$ ). It is known that cylindrical bluff body shape is more sensitive to vortex shedding than rectangular body. There are some studies on the vibration frequencies of the ski jumping skis, but they do not tell anything about the vortex shedding. During the flight phase the front and rear part of the jumping ski are oscillating as a cantilever beam fixed at the pivot near the balance point. Front part vibration is clearly seen in the supplementary video file of our manuscript. The first natural frequencies of the forebody and afterbody of jumping skis are lower than $10 \mathrm{~Hz}$. The second and third natural frequencies are about 25-35 and 70-90 Hz [10]. Videos from the present measurements show no steady frequencies for the vibration.

Based on earlier studies in ski jumping (e.g., [4]), the essential region of the AoA for the lift-to-drag ratio is 20-30 (early flight phase, low $C_{D}$ area in Fig. 3c), and $30-40^{\circ}$ for $C_{L}$ (latter part of flight phase). It seems that small edge angles $\left(0^{\circ}, 5^{\circ}\right.$ and $\left.10^{\circ}\right)$ at a typical yaw angle of $15^{\circ}$ don't dramatically affect $C_{L}(0.90,0.88,0.87$, respectively), while larger angles of $20^{\circ}, 30^{\circ}$ and $45^{\circ}$ decrease it considerably $(0.61,049,0.32$, respectively) (Figs. 3b, c, 4a). Regardless of the yaw angle, large edge 
Fig. 4 a The combined effect of yaw $\left(0^{\circ}, 2^{\circ}, 5^{\circ}, 10^{\circ}, 15^{\circ}\right.$ and $\left.20^{\circ}\right)$ and edge angles $\left(0^{\circ}, 5^{\circ}\right.$, $10^{\circ}, 20^{\circ}, 30^{\circ}$ and $45^{\circ}$ ) with a $30^{\circ} \mathrm{AoA}$ on $C_{L}$ and $\mathbf{b}$ on the emphasized lift-to-drag ratio $C_{L}^{2} / C_{D}$
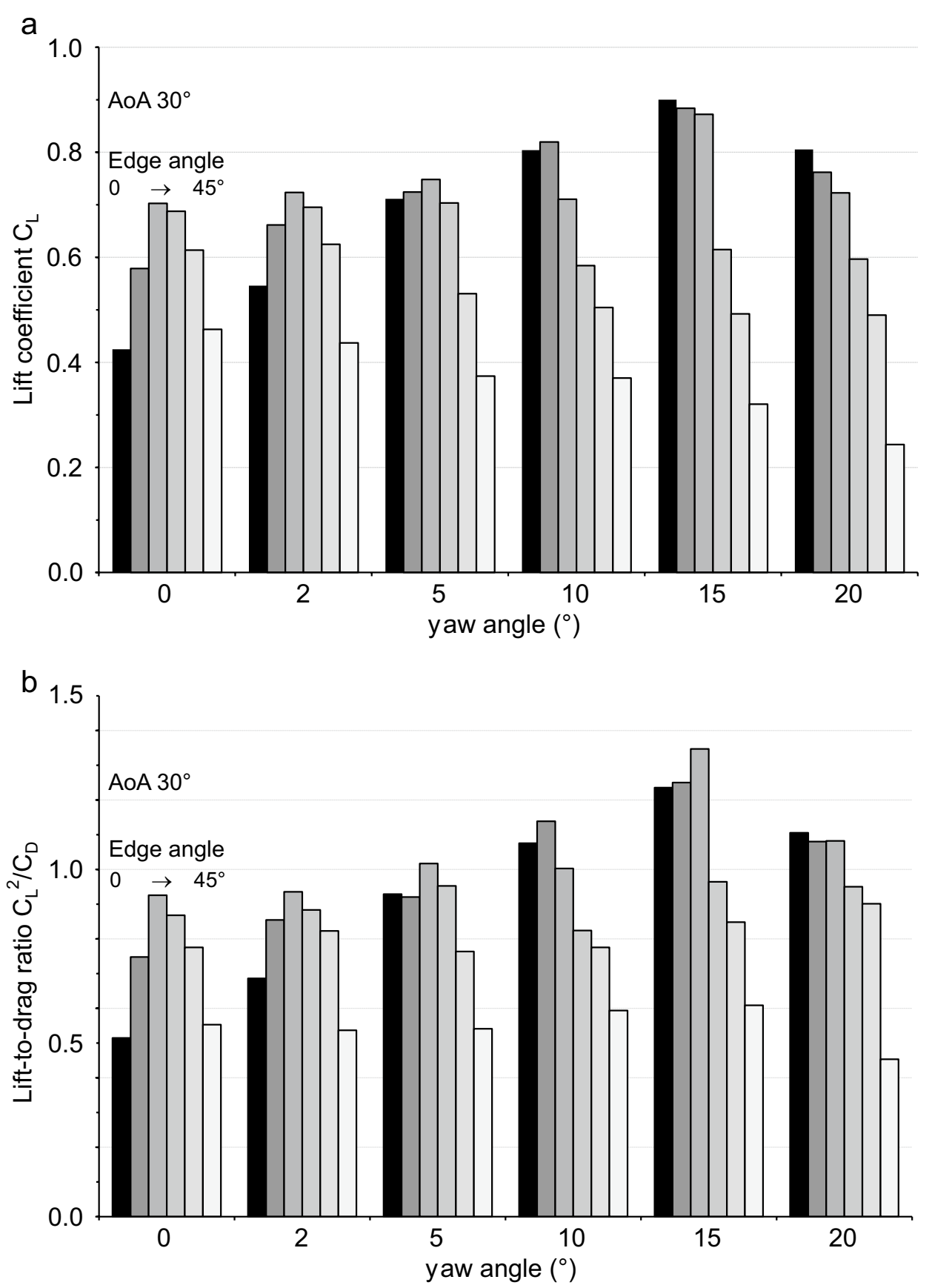

angles decrease the lift-to-drag ratio $\left(C_{L}^{2} / C_{D}\right)$, while an increase in yaw from zero up to $15^{\circ}$ at any given edge angle increases the ratio (e.g., from 0.51 to 1.24 at $0^{\circ}$ edge angle and 0.93 to 1.35 at $10^{\circ}$ edge angle) (Fig. 4b). The importance of $C_{L}$ is emphasized in Fig. 4b showing the product of $C_{L}$ and $C_{L} / C_{D}$ which also includes a lift-to-drag ratio. The results of this study show that the aerodynamics of an isolated ski in a wind tunnel depends strongly on the combined effect of the three angles varied in the present experiments (yaw, edge and AoA angles).
One way to estimate the effect of different aerodynamic factors (e.g., $C_{L}$ ) on jumping distance in ski jumping is to utilize a computer simulation. Sensitivity analysis based on the computer simulation shows that a $1 \%$ increase in $C_{L}$ during the entire flight phase of the reference jump $(134 \mathrm{~m})$ increases the jumping distance by $1.7 \mathrm{~m}$ [1]. Assuming the ski area represents only about half of the total aerodynamic area of the jumper/skis system [1], a small decrease in $C_{L}$ between the edge angles 0 and $10^{\circ}$ at yaw angle of $15^{\circ}$ (Fig. 4a) reduces jumping distance by $3 \mathrm{~m}$. However, this is a theoretical value assuming that the other factors remain same. In the present 


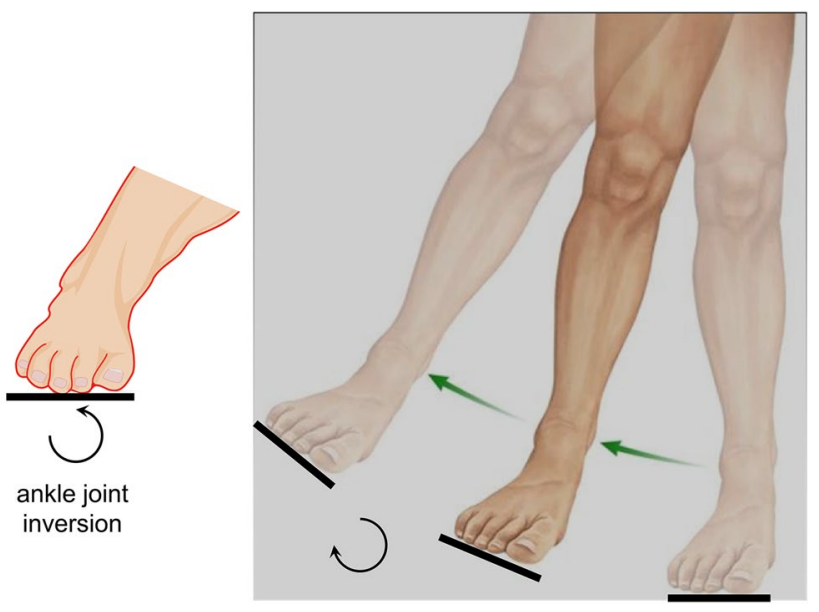

Fig. 5 Schematic illustration of the formation of the ski edge angle in $V$-style jumping (roll around the longitudinal axis of the ski) and possible ankle joint inversion to compensate for the roll (left)

set-up of isolated ski, the AoA, yaw and edge angles are so closely linked together that the above-mentioned loss in jumping distance can be at least partly compensated by the decreased $C_{D}$ seen in the lift-to-drag ratio in Fig. $4 \mathrm{~b}$.

The applicability of the present results to a complete jumper/ski system in actual jumping hill conditions is difficult to assess. As a $V$-style ski jumper spreads the skis out from the body, the jumper and the skis represent (in a simplified form) three lifting bodies, and each part has a similar wake system [6]. Therefore, the air flows around the skis and the jumper's body are more or less independent of each other, so the results of the present study may apply to jumping. The situation may be somewhat different if the skis are very close to the body, forming almost one plane (flat $V$-style, [11]). Jin et al. [11] found that the aerodynamic characteristics of flat $V$-style are better than $V$-style where the skis are not so close to the body. Nørstrud and Øye [6] described the trailing vortices behind three lifting plates representing ski jumper and skis and because the mentioned vortex systems are connected to up- and downwash, the authors mentioned that it is necessary to separate the bodies apart.

As mentioned at the end of the introduction the effect of the curved sticks that are used by ski jumpers in their ski bindings (Fig. 1b) to keep skis more flat may be overestimated. The curved sticks are designed to minimize the edge angle as the skis tend to follow the soles of the feet when the skis are set to the $V$ position (Fig. 5). Figure 5 also illustrates a jumpers' possibility to turn the skis to a more flat position by inversion at the ankle joint. Cutter [7] considered that an ankle angle of $20^{\circ}$ was the maximum feasible inversion for a ski jumper. Turning the skis more flat against the direction of motion may increase the positive pitching moment, which in turn may affect the stability of the flight.
It can be concluded that increasing yaw angle ( $V$ angle) increased the sensitivity of the ski to changes in edge angle, i.e., increasing the edge angle $\left(20^{\circ}-45^{\circ}\right)$ decreased the $C_{L}$ and the ratio $C_{L}^{2} / C_{D}$ with large yaw angles. As seen in Fig. $4 \mathrm{~b}$, to maximize the lift-to-drag ratio with an AoA in the region of $30^{\circ}$, it may be reasonable to have an edge angle of $5^{\circ}-10^{\circ}$ on skis.

Acknowledgements Open access funding provided by University of Jyväskylä (JYU).

\section{Compliance with ethical standards}

Conflict of interest The authors declare that they have no conflict of interest.

Open Access This article is distributed under the terms of the Creative Commons Attribution 4.0 International License (http://creativeco mmons.org/licenses/by/4.0/), which permits unrestricted use, distribution, and reproduction in any medium, provided you give appropriate credit to the original author(s) and the source, provide a link to the Creative Commons license, and indicate if changes were made.

\section{References}

1. Virmavirta M (2016) Aerodynamics of ski jumping. In: Braghin et al. (eds.) The engineering approach to winter sports. Springer, New York pp 153-181

2. Mahnke R, Hochmuth G (1990) Neue Erkenntnisse zur Luftkraftwirkung beim Ski-springen. Research Report, Forschungsinstitut für Körperkultur und Sport, Leipzig

3. Seo K, Murakami M, Yoshida K (2004) Optimal flight technique for V-style ski jumping. Sports Eng 7:97-104

4. Jung A, Staat M, Müller W (2014) Flight style optimization in ski jumping on normal, large, and ski flying hills. J Biomech 47(3):716-722

5. Bessone V, Petrat J, Seiberl W, Schwirtz A (2018) Analysis of landing in ski jumping by means of inertial sensors and force insoles. In: Proceedings of the 12th conference of the international sports engineering association, Brisbane, Queensland, Australia, 2, 311; https://doi.org/10.3390/proceedings2060311

6. Nørstrud H, Øye IJ (2009) On CFD simulation of ski jumping, in computational fluid dynamics for sport simulation. In: Peters $\mathrm{M}$ (ed) Lecture notes in computational science and engineering, vol 72. Springer, Berlin, pp 63-82

7. Cutter DA (1993) AIAA, aeronautical engineering, United States air force academy. Colorado Springs, Colorado

8. FIS (2017) Specifications for Competition Equipment and Commercial Markings. http://www.fis-ski.com. Accessed 18 Jun 2017

9. Krause E (2005) Wind tunnel for low speeds (Göttingen-type wind tunnel). Fluid mechanics: with problems and solutions, and an aerodynamics laboratory. Springer, Berlin, pp 233-243

10. Gim J, Jeon J, Kim B, Jeong T, Jeon K, Rhee B (2017) Quantification and design of jumping-ski characteristics. J Sports Eng Technol 1:1. https://doi.org/10.1177/1754337117721831

11. Jin H, Shimizu S, Watanuki T, Kubota H, Kobayashi K (1995) Desirable gliding styles and techniques in ski jumping. J Appl Biomech 11:460-474 